\title{
THE TOEPLITZ OPERATOR PROOF OF NONCOMMUTATIVE BOTT PERIODICITY
}

\author{
N. CHRISTOPHER PHILLIPS
}

(Received 20 April 1990)

Communicated by C. Sutherland

\begin{abstract}
We adapt the Toeplitz operator proof of Bott periodicity to give a short direct proof of Bott periodicity for the representable $K$-theory of $\sigma-C^{*}$-algebras. We further show how the use of this proof and the right definitions simplifies the derivation of the basic properties of representable $K$-theory.
\end{abstract}

1991 Mathematics subject classification (Amer. Math. Soc.): primary 19 K 99, 46 K 99, 46 L 80; secondary $19 \mathrm{~K} 56,46 \mathrm{M} 20,46 \mathrm{M} 40$.

\section{Introduction}

In this paper, we give a short and direct proof of Bott periodicity for the representable $K$-theory of $\sigma-C^{*}$-algebras [12], using the Toeplitz operator method of [2]. We use definitions for representable $K$-theory which first appeared in [13] and which are closely related to the usual definition of $K$-theory for $C^{*}$-algebras, and our proof is not much more complicated than it would be for $C^{*}$-algebras. Thus, this paper can also be regarded as an exposition of the Toeplitz operator proof of Bott periodicity for the $K$-theory of $C^{*}$-algebras. We also include direct proofs that our definitions of representable $K$-theory agree with the usual $K$-theory of $C^{*}$-algebras, and show how the definitions

This research was partially supported by an NSF postdoctoral fellowship and by NSF grant no. DMS 86-03223.

(C) 1992 Australian Mathematical Society $0263-6115 / 92 \$ A 2.00+0.00$ 
we use simplify the proofs of the basic properties of representable $K$-theory.

The literature contains two elementary proofs of Bott periodicity for the $K$-theory of compact spaces, one involving approximation arguments on clutching functions [3] and one by Atiyah using Toeplitz operators [2]. The first of these proofs generalizes nicely to the $K$-theory of Banach algebras: see the exposition in [19, Section 9]. The resulting proof, however, seems unnatural for $C^{*}$-algebras, because it uses approximation arguments on invertible elements that take one out of the group of unitary elements of a $C^{*}$-algebra. The Toeplitz operator proof, on the other hand, can be done for $C^{*}$-algebras using only unitaries and projections. This proof is well known, but the $C^{*}$-algebra case has apparently never been published. The closest approximation we know in the literature is due to Cuntz [5]. That version is short, slick, and applies to a more general situation, but obscures the essential simplicity of the ideas involved. Section 3 of this paper gives essentially the noncommutative version of Atiyah's Toeplitz operator proof, including a remark about the simplification possible when one considers $C^{*}$-algebras instead of $\sigma-C^{*}$-algebras.

We now turn to a $\sigma-C^{*}$-algebras. Two proofs of Bott periodicity for representable $K$-theory have appeared: one in Weidner's thesis [20] (see also [21]), which actually applies much more generally but uses much complicated machinery, and one in [12], which is somewhat indirect, using the Milnor $\lim ^{1}$ sequence to reduce to the case of $C^{*}$-algebras. The approximation proof in [19, Section 9] does not generalize to $\sigma-C^{*}$-algebras, because the group of invertible elements in a unital $\sigma-C^{*}$-algebra is not open (see [11, Proposition 1.14]). However, as we show, the Toeplitz operator proof, which uses only unitaries and projections, generalizes very nicely to the $\sigma-C^{*}$-algebra case, provided that one uses the right definitions.

The definitions we use are taken from [13]. We believe that these definitions are the right ones to use, and as evidence for that belief we offer their obvious connection with the usual definitions of $K$-theory for $C^{*}$-algebras, the fact that they work well in the proof given here, the ease with which the main properties of representable $K$-theory are derived from them, and the fact that they generalize to Fréchet algebras ([16]). We show in Section 4 of this paper how the development of [12] is simplified if one uses the definitions of this paper.

The proof of Bott periodicity given here also serves two other purposes. One is to provide a proof of Bott periodicity which generalizes to Fréchet algebras. As demonstrated in [16], the proof given here does so very nicely; as far as we know, no other proof does. For the second purpose, recall that the main theorem of [14] asserted the existence of homotopy equivalences 
$\Omega_{\infty} U_{\mathrm{nc}} \simeq P$ and $\Omega_{\infty} P \simeq U_{\mathrm{nc}}$ involving the $C^{\infty}$ loop algebras of the representing algebras $P$ and $U_{\text {nc }}$ for $K$-theory. (The connection between this result and Bott periodicity is explained in [15].) The proof given there was very indirect, relying eventually on the Bott periodicity theorem in [12]. We plan, in a future paper, to give direct proofs of these homotopy equivalences, and our proposed direct proofs are closely modelled on the proofs in this paper. Partly because of this application, we avoid Atiyah's trick ([1, Section 1]) in our proof, giving instead at the appropriate point a direct calculation.

For readers interested only in $C^{*}$-algebras, the logical prerequisites of this paper are only the standard definitions of $K_{0}(A)$ and $K_{1}(A)$ in terms of projections and unitaries. (See for example Blackadar's book [4].) For readers interested in $\sigma \cdot C^{*}$-algebras, some of their basic properties are needed; see [11, Sections 1,3 , and 5]. We also need the $\sigma-C^{*}$-algebra versions of two basic lemmas on unitaries and projections, which appear as isolated lemmas in two of our earlier papers; these are stated at the end of Section 1.

This paper is organized as follows. In Section 1, we state the definitions of $K$-theory that we use, prove from scratch that they agree with the usual groups for a $C^{*}$-algebra, and state several necessary lemmas. Sections 2 and 3 contain the proofs of the "easy" and "hard" halves of Bott periodicity, including, in Section 3, an explanation of the simplifications that appear in the $C^{*}$-algebra case. Section 4 shows how to simplify the derivation of the basic properties of representable $K$-theory, including the equivariant case, by using the approach of the first three sections.

\section{Preliminaries}

In this section, we give our definitions of representable $K$-theory, and prove from scratch that they give the usual groups for $C^{*}$-algebras. We also give the analogs for $\sigma$ - $C^{*}$-algebras of several standard lemmas on $C^{*}$ algebras.

Throughout this paper, $K$ is the algebra of compact operators on a fixed separable infinite dimensional Hilbert space, and $A^{+}$is the unitization of a $\sigma-C^{*}$-algebra $A$. If $A$ is unital, then $U(A)$ is the unitary group and $U_{0}(A)$ is the path component of 1 in $U(A)$.

1.1 Definition. Let $A$ be a $\sigma-C^{*}$-algebra. Then $R K_{0}(A)$ is the set of homotopy classes in the set of projections $p \in M_{2}\left((K \otimes A)^{+}\right)$such that $p-\left(\begin{array}{ll}1 & 0 \\ 0 & 0\end{array}\right) \in M_{2}(K \otimes A)$. Also, $R K_{1}(A)$ is the set of homotopy classes in the set of unitaries $u \in(K \otimes A)^{+}$such that $u-1 \in K \otimes A$.

In both sets, we define addition to be direct sum, followed for $R K_{0}$ by 
conjugation by the unitary

$$
c=\left[\begin{array}{llll}
1 & 0 & 0 & 0 \\
0 & 0 & 1 & 0 \\
0 & 1 & 0 & 0 \\
0 & 0 & 0 & 1
\end{array}\right],
$$

followed in both cases by an identification of $M_{2}(K)$ with $K$.

If $\varphi: A \rightarrow B$ is a homomorphism of $\sigma-C^{*}$-algebras, then we define $\varphi_{*}: R K_{i}(A) \rightarrow R K_{i}(B)$ by $\varphi([p])=\left[\left(\operatorname{id}_{M_{2}(K)} \otimes \varphi\right)^{+}(p)\right]$ for $i=0$ and $\varphi([u])=\left[\left(\mathrm{id}_{K} \otimes \varphi\right)^{+}(u)\right]$ for $i=1$.

The definition of $R K_{0}$ is taken from [13, Definition 2.2]; note that $c$ is chosen so as to conjugate $\left(\begin{array}{ll}1 & 0 \\ 0 & 0\end{array}\right) \oplus\left(\begin{array}{ll}1 & 0 \\ 0 & 0\end{array}\right)$ to $1 \oplus 0$. The definition of $R K_{1}$ is suggested by [13, Definition 3.3], with modifications to make it more closely resemble the definition of $R K_{0}$. Note that we do not need separate definitions for nonunital $A$. This simplifies the bookkeeping in some proofs.

1.2 RemarK. We will use $K_{0}(A)$ and $K_{1}(A)$ to refer to the $K$-theory of a $C^{*}$-algebra $A$, defined as in, for example, [4, Sections 5.5 and 8.1]. In the definition of $K_{0}(A)$, we will use projections and either unitary equivalence or homotopy as appropriate (see [4, Section 4.6]).

It follows from [13] and [12] that $R K_{*}$ as defined here is the same as in our earlier papers. It therefore follows that $R K_{i}(A) \cong K_{i}(A)$ for $C^{*}$-algebras $A$. We will, however, give a selfcontained proof in this section. Note that the definition of $R K_{1}(A)$ is very close to the usual definition of $K_{1}(A)$ for $A$ unital. The definition of $R K_{0}(A)$ is not quite so close to the usual definition of $K_{0}(A)$, but the examples in [12, Section 4] show that one can't expect to do better.

For completeness, we show here that $R K_{0}(A)$ and $R K_{1}(A)$ really are functorial abelian groups.

1.3 LemmA. $R K_{0}(A)$ and $R K_{1}(A)$ are abelian semigroups with identities $\left[\left(\begin{array}{ll}1 & 0 \\ 0 & 0\end{array}\right)\right]$ and [1]. The map $\varphi_{*}$ is a well defined semigroup homomorphism which depends only on the homotopy class of $\varphi$.

Proof. It is immediate that addition in both sets is well defined. (It does not depend on the choice of the isomorphism $M_{2}(K) \cong K$, because any two are homotopic.) That the identities are as claimed is proved by using a homotopy from an isomorphism $K \rightarrow M_{2}(K)$ to the inclusion $a \mapsto a \oplus 0$ of $K$ in $M_{2}(K)$. Associativity is proved similarly. Commutativity is obtained via the standard homotopy from $a \oplus b$ to $b \oplus a$. The statements about $\varphi_{*}$ are trivial. 
1.4 Proposition. $R K_{0}(A)$ is a functorial homotopy invariant abelian group.

Proof. It only remains to prove that inverses exist. We claim that $-[p]=$ $\left[\left(\begin{array}{ll}0 & 1 \\ 1 & 0\end{array}\right)\left(\begin{array}{ll}1 & -p\end{array}\right)\left(\begin{array}{ll}0 & 1 \\ 1 & 0\end{array}\right)\right]$. Eliminating the conjugation by $c$ in the definition of addition, we see that it suffices to find a homotopy of projections from

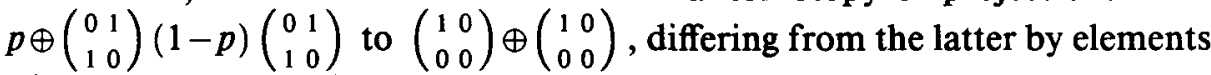
of $M_{4}(K \otimes A)$. Equivalently, we produce a homotopy from $p \oplus(1-p)$ to $\left(\begin{array}{ll}1 & 0 \\ 0 & 0\end{array}\right) \oplus\left(\begin{array}{ll}0 & 0 \\ 0 & 1\end{array}\right)$, differing from the latter by elements of $M_{4}(K \otimes A)$.

Let $k=\pi / 2$ and define

$$
w_{t}=\left[\begin{array}{cc|cc}
\cos (k t) & 0 & \sin (k t) & 0 \\
0 & \cos (k t) & 0 & \sin (k t) \\
\hline-\sin (k t) & 0 & \cos (k t) & 0 \\
0 & -\sin (k t) & 0 & \cos (k t)
\end{array}\right],
$$

which is a unitary path in $M_{4}(\mathbb{C})$ from $w_{0}=1$ to

$$
w_{1}=\left[\begin{array}{lllll}
0 & & 1 & 1 & 0 \\
& & 1 & 0 & 1 \\
\hline 1 & 0 & 1 & & \\
0 & 1 & 1 & & 0
\end{array}\right] .
$$

Then $w_{t}(0 \oplus(1-p)) w_{t}^{*}$ is orthogonal to $p \oplus 0$ for all $t$, and similarly with $\left(\begin{array}{ll}1 & 0 \\ 0 & 0\end{array}\right)$ in place of $p$. Choose a unitary path $t \mapsto x_{t}$ in $M_{4}(\mathbb{C})$ with $x_{0}=1$ and

$$
\text { (*) } \quad x_{t}\left[\left(\begin{array}{ll}
1 & 0 \\
0 & 0
\end{array}\right) \oplus 0+w_{t}\left[0 \oplus\left(\begin{array}{ll}
0 & 0 \\
0 & 1
\end{array}\right)\right] w_{t}^{*}\right] x_{t}^{*}=\left(\begin{array}{ll}
1 & 0 \\
0 & 0
\end{array}\right) \oplus\left(\begin{array}{ll}
0 & 0 \\
0 & 1
\end{array}\right) .
$$

(See the $C^{*}$-algebra version of Lemma 1.9 below.) Define

$$
q_{t}=x_{t}\left[p \oplus 0+w_{t}(0 \oplus(1-p)) w_{t}^{*}\right] x_{t}^{*} .
$$

Then $q_{0}=p \oplus(1-p), q_{1}=\left(\begin{array}{ll}1 & 0 \\ 0 & 0\end{array}\right) \oplus\left(\begin{array}{ll}0 & 0 \\ 0 & 1\end{array}\right)$ (using $(*)$ at $t=1$ ), and it follows from $(*)$ that $q_{t}-\left(\begin{array}{ll}1 & 0 \\ 0 & 0\end{array}\right) \oplus\left(\begin{array}{ll}0 & 0 \\ 0 & 1\end{array}\right) \in M_{4}(K \otimes A)$ for all $t$. Therefore $t \mapsto q_{t}$ is the required homotopy.

1.5 Proposition. $R K_{1}(A)$ is a functorial homotopy invariant abelian group, in which $[u]+[v]=[u v]$.

Proof. We only have to show that $[u]+[v]=[u v]$ for $u, v \in U\left((K \otimes A)^{+}\right)$ with $u-1, v-1 \in K \otimes A$. It suffices to prove that $[u \oplus v]=[u v \oplus 1]$, since we already know that [1] is the identity. The standard construction yields a 
homotopy $t \mapsto z_{t}$ from $u \oplus v$ to $u v \oplus 1$ which satisfies $z_{t}-1 \in M_{2}(K \otimes A)$ for all $t$.

We now prove the isomorphisms $R K_{i}(A) \cong K_{i}(A)$ for $C^{*}$-algebras.

1.6 Proposition. For any $C^{*}$-algebra $A$, there is a natural isomorphism $R K_{0}(A) \cong K_{0}(A)$.

Proof. Let $p_{0}$ denote the projection $\left(\begin{array}{ll}1 & 0 \\ 0 & 0\end{array}\right)$, in the $2 \times 2$ matrices over any unital $C^{*}$-algebra. Stability and the split exact sequence

$$
0 \rightarrow K \otimes A \rightarrow(K \otimes A)^{+} \stackrel{\pi}{\rightarrow} \mathbb{C} \rightarrow 0
$$

give an isomorphism $K_{0}(A) \cong \operatorname{Ker}\left(\pi_{*}\right) \subset K_{0}\left((K \otimes A)^{+}\right)$. Define $\Phi: R K_{0}(A)$ $\rightarrow \operatorname{Ker}\left(\pi_{*}\right)$ by $\Phi([p])=[p]-\left[p_{0}\right]$. Then $\Phi$ is well defined because homotopic projections are equivalent, and the image of $\Phi$ really is in $\operatorname{Ker}\left(\pi_{*}\right)$ because $M_{2}(\pi)(p)=p_{0} \in M_{2}(\mathbb{C})$. We now show that $\Phi$ is a homomorphism. Let $\imath: \mathbb{C} \rightarrow M_{2}(\mathbb{C})$ be the inclusion in the upper left corner. Note that $l$ determines a homomorphism $\left(l \otimes \mathrm{id}_{A}\right)^{*}: R K_{0}(A) \rightarrow R K_{0}\left(M_{2}(A)\right)$ and an isomorphism $\left(l \otimes \mathrm{id}_{K \otimes A}\right)^{*}: K_{0}(K \otimes A) \rightarrow K_{0}\left(M_{2}(K \otimes A)\right)$ such that $\left(l \otimes \mathrm{id}_{K \otimes A}\right)^{*} \circ \Phi_{A}=\Phi_{M_{2}(A)} \circ\left(l \otimes \mathrm{id}_{A}\right)^{*}$. Therefore it suffices to show that

$(*) \quad \Phi_{M_{2}(A)} \circ\left(l \otimes \mathrm{id}_{A}\right)^{*}\left(\left[p_{1}\right]+\left[p_{2}\right]\right)=\left(l \otimes \mathrm{id}_{K \otimes A}\right)^{*}\left(\Phi\left(\left[p_{1}\right]\right)+\Phi\left(\left[p_{2}\right]\right)\right)$.

Let $\varphi: M_{2}(K) \rightarrow K$ be an isomorphism, and let $c$ be as in Definition 1.1, so that $\left[p_{1}\right]+\left[p_{2}\right]=\left[M_{2}\left(\left(\varphi \otimes \mathrm{id}_{A}\right)^{+}\right)\left(c\left(p_{1} \oplus p_{2}\right) c^{*}\right)\right]$. Now $\left(l \otimes \mathrm{id}_{K}\right) \circ \varphi$ is homotopic to $\mathrm{id}_{M_{2}(K)}$. Therefore the left hand side of $(*)$ is $\left[c\left(p_{1} \oplus p_{2}\right) c^{*}\right]-\left[c\left(p_{0} \oplus p_{0}\right) c^{*}\right]$. Working in $M_{4}\left((K \otimes A)^{+}\right)$, we see that this is equal to $\left[p_{1} \oplus p_{2}\right]-\left[p_{0} \oplus p_{0}\right]$, which in turn is equal to the left hand side of $(*)$.

To check that $\Phi$ is surjective, represent an arbitrary class $\eta \in K_{0}(A)$ as $[p]-\left[p_{0}\right]$, where $p=\left(\begin{array}{l}p_{11} p_{12} \\ p_{21}\end{array} p_{22}\right) \in M_{2}\left(M_{n}\left(A^{+}\right)\right)$and $p-p_{0} \in M_{2}\left(M_{n}(A)\right)$. (See the remark following [4, Definition 5.5.1].) Then $e=\left(\begin{array}{cc}p_{11} \oplus 1 & p_{12} \\ p_{21} & p_{22}\end{array}\right)$ is a projection in $M_{2}\left((K \otimes A)^{+}\right)$with $e-p_{0} \in M_{2}(K \otimes A)$, and $\Phi([e])=\eta$.

Finally, we prove that $\Phi$ is injective. Let $\Phi([p])=0$. Then in some $M_{n}\left(M_{2}\left((K \otimes A)^{+}\right)\right)$, there is a homotopy $t \mapsto q_{t}$ from $q_{0}=p \oplus p_{0} \oplus \cdots \oplus$ $p_{0}$ to $q_{1}=p_{0} \oplus \cdots \oplus p_{0}$ ( $n$ summands in both expressions). Let $\pi$ : $M_{2 n}\left((K \otimes A)^{+}\right) \rightarrow M_{2 n}(\mathbb{C})$ be the quotient map. Find a continuous unitary path $t \mapsto u_{t}$ in $M_{2 n}(\mathbb{C})$ with $u_{0}=1$ and $u_{t} \pi\left(q_{t}\right) u_{t}^{*}=\pi\left(q_{0}\right)$. (Note

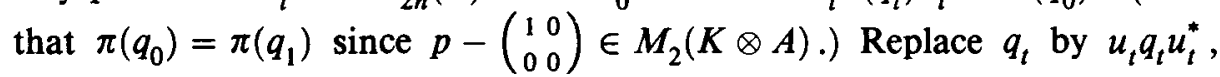
regarding $u_{t}$ as being in $M_{2 n}\left((K \otimes A)^{+}\right)$. This does not change either $q_{0}$ or 
$q_{1}$, but now $q_{t}-p_{0} \oplus \cdots \oplus p_{0} \in M_{2 n}(K \otimes A)$ for all $t$. This homotopy now shows that $[p]+(n-1)\left[p_{0}\right]=0$ in $R K_{0}(A)$. So $[p]=0$.

1.7 Proposition. For any $C^{*}$-algebra $A$, there is a natural isomorphism $R K_{1}(A) \cong K_{1}(A)$.

Proof. See the remarks following [4, Definition 8.1.1].

The following two lemmas are the analogs for $\sigma-C^{*}$-algebras of two lemmas which are important in the $K$-theory of $C^{*}$-algebras. We state them here for ease of reference, but refer to our earlier papers for the proofs. It should be noted that the proofs do not depend on the theory developed in those papers.

1.8 Lemma ([12, Lemma 1.11]). Let $A \rightarrow B$ be a surjective homomorphism of unital $\sigma-C^{*}$-algebras. Then $U_{0}(A) \rightarrow U_{0}(B)$ is surjective.

1.9 Lemma ([13, Lemma 2.4]). Let $A$ be a unital $\sigma-C^{*}$-algebra, and let $t \mapsto p_{t}$ be a continuous path of projections in $A$. Then there is a continuous path $t \mapsto u_{t}$ of unitaries in $A$ such that $u_{0}=1$ and $u_{t} p_{0} u_{t}^{*}=p_{t}$. If $p_{t}-p_{0}$ is in an ideal $I$ for all $t$, then we can take $u_{t}-1 \in I$.

Finally, we record here a fact which has been used implicitly elsewhere and will be needed later in this paper. Call a $\sigma-C^{*}$-algebra nuclear if it is the inverse limit of a system of nuclear $C^{*}$-algebras with surjective maps, as in the remarks following [11, Proposition 3.3].

1.10 Lemma. Let $0 \rightarrow I \rightarrow A \rightarrow B \rightarrow 0$ be an exact sequence of $\sigma$ $C^{*}$-algebras, and let $D$ be a $\sigma$ - $C^{*}$-algebra. If $A$ or $D$ is nuclear, then $0 \rightarrow I \otimes D \rightarrow A \otimes D \rightarrow B \otimes D \rightarrow 0$ is exact.

Proof. Write the exact sequence $0 \rightarrow I \rightarrow A \rightarrow B \rightarrow 0$ as an inverse limit of exact sequences $0 \rightarrow I_{n} \rightarrow A_{n} \rightarrow B_{n} \rightarrow 0$ of $C^{*}$-algebras, with all maps in the inverse systems being surjective ([11, Proposition 5.3(2)]). Also write $D=\lim _{n} D_{n}$ with all maps surjective. Then each sequence $0 \rightarrow I_{n} \otimes D_{n} \rightarrow$ $A_{n} \otimes \overleftarrow{D}_{n} \rightarrow B_{n} \otimes D_{n} \rightarrow 0$ is exact, since $A_{n}$ or $D_{n}$ is nuclear. Also the maps $I_{n+1} \otimes D_{n+1} \rightarrow I_{n} \otimes D_{n}$, etc., are all surjective, so by [11, Proposition 5.3(2)], the inverse limit $0 \rightarrow I \otimes D \rightarrow A \otimes D \rightarrow B \otimes D \rightarrow 0$ of these sequences is exact. 


\section{The Isomorphism of $K_{0}(S A)$ with $K_{1}(A)$}

In this section we prove the easy half of Bott periodicity. No Toeplitz operators will appear here.

In the $K$-theory of $C^{*}$-algebras, this part is usually obtained from the exact sequence

$$
K_{1}(I) \rightarrow K_{1}(B) \rightarrow K_{1}(B / I) \stackrel{\partial}{\rightarrow} K_{0}(I) \rightarrow K_{0}(B) \rightarrow K_{0}(B / I)
$$

corresponding to an exact sequence $0 \rightarrow I \rightarrow B \rightarrow B / I \rightarrow 0$. One sets $I=S A=C_{0}((0,1)) \otimes A, B=C A=C_{0}([0,1)) \otimes A$, and $B / I=A$, and observes that $K_{0}(B)=K_{1}(B)=0$ because $B$ is contractible. (See for example [19, Section 8].) Here we will follow the approach used in the proof of [4, Theorem 8.2.2], and give a direct proof that $R K_{0}(S A) \cong R K_{1}(A)$. An examination of the connecting homomorphism in $(*)$ shows, however, that our proof is essentially the same as the one in [19].

2.1 Definition. Let $A$ be a $\sigma-C^{*}$-algebra. Define $\lambda: R K_{1}(A) \rightarrow R K_{0}(S A)$ as follows. Given $u \in U\left((K \otimes A)^{+}\right)$with $u-1 \in K \otimes A$, choose some continuous path $t \mapsto v(t)$ in $U\left(M_{2}\left((K \otimes A)^{+}\right)\right)$with $v(0)=\left(\begin{array}{ll}1 & 0 \\ 0 & 1\end{array}\right), v(1)=\left(\begin{array}{ll}u & 0 \\ 0 & x\end{array}\right)$ for some $x$, and $v(t)-\left(\begin{array}{ll}1 & 0 \\ 0 & 1\end{array}\right) \in M_{2}(K \otimes A)$ for all $t$. (For example, we could take

$$
v(t)=\left(\begin{array}{cc}
u & 0 \\
0 & 1
\end{array}\right)\left(\begin{array}{cc}
\cos (k t) & \sin (k t) \\
-\sin (k t) & \cos (k t)
\end{array}\right)\left(\begin{array}{cc}
u^{*} & 0 \\
0 & 1
\end{array}\right)\left(\begin{array}{cc}
\cos (k t) & \sin (k t) \\
-\sin (k t) & \cos (k t)
\end{array}\right)^{*},
$$

with $k=\pi / 2$, giving $x=u^{*}$.) Define $p(t)=v(t)\left(\begin{array}{ll}1 & 0 \\ 0 & 0\end{array}\right) v(t)^{*}$. As we show in Lemma 2.3 below, $p$ defines a class in $R K_{0}(S A)$, and we take $\lambda([u])$ to be that class.

Define $\nu: R K_{0}(S A) \rightarrow R K_{1}(A)$ as follows. Let $p \in M_{2}\left((K \otimes S A)^{+}\right)$be a projection such that $p-\left(\begin{array}{ll}1 & 0 \\ 0 & 0\end{array}\right) \in M_{2}(K \otimes S A)$. Regard $p$ as a function from $[0,1]$ to $M_{2}\left((K \otimes A)^{+}\right)$, with $p(0)=p(1)=\left(\begin{array}{ll}1 & 0 \\ 0 & 0\end{array}\right)$ and $p(t)-\left(\begin{array}{ll}1 & 0 \\ 0 & 0\end{array}\right) \epsilon$ $M_{2}(K \otimes A)$. Use Lemma 1.9 to find a unitary path $t \mapsto v(t)$ in $M_{2}\left((K \otimes A)^{+}\right)$ such that $v(0)=\left(\begin{array}{ll}1 & 0 \\ 0 & 1\end{array}\right), v(t)\left(\begin{array}{ll}1 & 0 \\ 0 & 0\end{array}\right) v(t)^{*}=p(t)$, and $v(t)-\left(\begin{array}{ll}1 & 0 \\ 0 & 1\end{array}\right) \in M_{2}(K \otimes$ $A)$. Then $v(1)=\left(\begin{array}{ll}u & 0 \\ 0 & x\end{array}\right)$ for some unitaries $u, x \in(K \otimes A)^{+}$, and we define $\nu([p])$ to be the class of $u$ in $R K_{1}(A)$.

The following lemma is needed to prove that $\lambda$ is well defined.

2.2 LEMMA. Let $A$ be a pro- $C^{*}$-algebra, let $u \in U\left((K \otimes A)^{+}\right)$satisfy $u-1 \in K \otimes A$, and let $t \mapsto v_{0}(t)$ and $t \mapsto v_{1}(t)$ be two unitary paths 
in $M_{2}\left((K \otimes A)^{+}\right)$with $v_{i}(0)=\left(\begin{array}{ll}1 & 0 \\ 0 & 1\end{array}\right), v_{i}(1)=\left(\begin{array}{ll}u & 0 \\ 0 & x_{i}\end{array}\right)$, and $v_{i}(t)-\left(\begin{array}{ll}1 & 0 \\ 0 & 1\end{array}\right) \in$ $M_{2}(K \otimes A)$. Then there exists a continuous function $(s, t) \mapsto v_{s}(t) \in$ $U\left(M_{2}\left((K \otimes A)^{+}\right)\right)$with $v_{s}(t)$ as given for $s=0$ and $s=1$, with $v_{s}(1)=$ $\left(\begin{array}{ll}u & 0 \\ 0 & x_{s}\end{array}\right)$, and with $v_{s}(t)-\left(\begin{array}{ll}1 & 0 \\ 0 & 1\end{array}\right) \in M_{2}(K \otimes A)$ for all $s, t \in[0,1]^{2}$.

Proof. Multiplying $v_{s}(t)$ by $v_{0}(t)^{*}$ for $s=0,1$, we reduce to the case in which $u=1$ and $v_{0}(t)=1$ for all $t$. Now let $\varphi: M_{2}(K \otimes A)^{+} \rightarrow(K \otimes A)^{+}$be an isomorphism determined by an isomorphism $\varphi_{0}: M_{2}(K) \rightarrow K$. Note that id $_{M_{2}(K)}$ is homotopic to $k \mapsto 0 \oplus \varphi_{0}(k)$, and furthermore that the homotopy can be chosen to send $0 \oplus K$ into $0 \oplus K$ (as subsets of $M_{2}(K)$ ) at every stage. This homotopy gives a homotopy $(s, t) \mapsto \bar{v}_{s}(t)$ from $t \mapsto v_{1}(t)$ to $t \mapsto 1 \oplus \varphi\left(v_{1}(t)\right)$. The condition on $0 \oplus K$ ensures that $\bar{v}_{s}(1)$ has the form $1 \oplus \bar{x}_{s}$ for every $s$. (Thus, $s \mapsto \bar{x}_{s}$ is a homotopy from $x_{1}$ to 1.) The other conditions of the lemma are trivially satisfied for the homotopy $s \mapsto \bar{v}_{s}$, and thus we may assume, in addition to the above, that $v_{1}(t)=1 \oplus w(t)$ for some unitary function $w$ with $w(0)=1$ and $w(t)-1 \in K \otimes A$. Now we simply set $u_{s}(t)=1 \oplus w(s t)$, a homotopy which obviously satisfies the condition required and gives $v_{0}=1$ as required.

2.3 LemMA. The maps $\lambda$ and $\nu$ of Definition 2.1 are well defined natural group homomorphisms.

Proof. The only issue is well definedness. In the definition of $\lambda$, an easy calculation proves that $p(t)-\left(\begin{array}{ll}1 & 0 \\ 0 & 0\end{array}\right)$ is in $M_{2}(K \otimes A)$ for all $t$, and equal to 0 for $t=0,1$. It follows that $p \in M_{2}\left((K \otimes S A)^{+}\right)$and $p-\left(\begin{array}{ll}1 & 0 \\ 0 & 0\end{array}\right) \in$ $M_{2}(K \otimes S A)$, so that $p$ defines a class in $R K_{0}(S A)$. Next, if $v_{0}$ and $v_{1}$ are two choices for $v$, let $v_{s}(t)$ be as in the previous lemma, and observe that the homotopy $s \mapsto p_{s}=v_{s}\left(\begin{array}{ll}1 & 0 \\ 0 & 0\end{array}\right) v_{s}^{*}$ shows that $\lambda([u])$ does not depend on the choice of $v$. Finally, if $s \mapsto u_{s}$ is a homotopy, then we can regard it as an element $u \in U\left([K \otimes C([0,1]) \otimes A]^{+}\right)$such that $u-1 \in K \otimes C([0,1]) \otimes A$. Choose $v$ as in the definition, for this $u$; then the homotopy $s \mapsto p_{s}$, with $p_{s}(t)=v_{s}(t)\left(\begin{array}{ll}1 & 0 \\ 0 & 0\end{array}\right) v_{s}(t)^{*}$, shows that $\lambda\left(\left[u_{0}\right]\right)=\lambda\left(\left[u_{1}\right]\right)$. This completes the proof that $\lambda$ is well defined.

Now we show that $\nu$ is well defined. It is clear that $u-1 \in K \otimes A$ in the definition, so that $u$ defines an element of $R K_{1}(A)$. Now let $v_{0}$ and

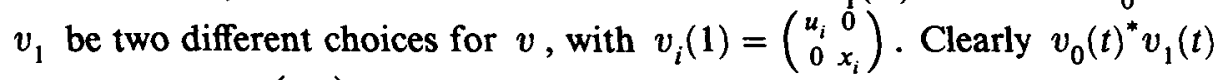
commutes with $\left(\begin{array}{ll}1 & 0 \\ 0 & 0\end{array}\right)$, and so has the form $t \mapsto y(t) \oplus z(t)$. Then $t \mapsto u_{0} y(t)$ is a homotopy from $u_{0}$ to $u_{1}$; thus, $\left[u_{0}\right]=\left[u_{1}\right]$, and $\nu([p])$ does not depend 
on $v$. That $\nu([p])$ depends only on the homotopy class of $p$ is now proved just as for $\lambda$.

2.4 THEOREM. There is a natural isomorphism $R K_{0}(S A) \cong R K_{1}(A)$.

Proof. We only have to prove that $\lambda \circ \nu$ and $\nu \circ \lambda$ are the identity maps. To do so, simply use the same $v$ in both parts of Definition 2.1.

\section{The Isomorphism of $K_{1}(S A)$ with $K_{0}(A)$}

Here we prove the more difficult half of Bott periodicity, using Toeplitz operators. In this section we take the suspension $S A$ to be $C_{0}\left(S^{1}-\{1\}\right) \otimes A$, regarded as a subalgebra of $C\left(S^{1}\right) \otimes A$ in the obvious way, where $S^{1}$ is the unit circle in $\mathbb{C}$. Also, $z$ will denote the function $z(\zeta)=\zeta$, regarded as an element of $C\left(S^{1}\right)$ or of $L^{2}\left(S^{1}\right)$.

3.1 Definition. Let $A$ be a $\sigma$ - $C^{*}$-algebra. Define $\beta: R K_{0}(A) \rightarrow$ $R K_{1}(S A)$ as follows. Set $p_{0}=\left(\begin{array}{ll}1 & 0 \\ 0 & 0\end{array}\right) \in M_{2}\left((K \otimes A)^{+}\right)$. For $p \in$ $M_{2}\left((K \otimes A)^{+}\right)$with $p-p_{0} \in M_{2}(K \otimes A)$, define $u \in U\left(M_{2}(K \otimes S A)^{+}\right)$ by $u(\zeta)=(1-p+\zeta p)\left(1-p_{0}+\zeta p_{0}\right)^{*}$. Identify $M_{2}(K \otimes S A)^{+}$with $(K \otimes S A)^{+}$ via some isomorphism $M_{2}(K) \rightarrow K$, and define $\beta([p])$ to be the class of $u$ in $R K_{1}(S A)$.

Before defining the inverse map, we introduce some notation. Let $\mu_{0}$ be the representation of $C\left(S^{1}\right)$ on $L^{2}=L^{2}\left(S^{1}\right)$ by multiplication operators, and let $\mu$ denote the obvious extension to a homomorphism from $C\left(S^{1}\right) \otimes$ $(K \otimes A)^{+}$to the multiplier algebra $M\left(K\left(L^{2}\right) \otimes(K \otimes A)^{+}\right)$. Let $q_{0}$ be the projection from $L^{2}$ to the Hardy space $H^{2}$, which is the closed linear span of the functions $z^{n}$ for $n \geq 0$. Also let $q=q_{0} \otimes 1$ be the corresponding projection in $M\left(K\left(L^{2}\right) \otimes(K \otimes A)^{+}\right)$. Using some isomorphism of $\left(H^{2}\right)^{\perp}$ with $H^{2}$, we identify $K\left(L^{2}\right) \otimes(K \otimes A)^{+}$with $M_{2}\left(K\left(H^{2}\right) \otimes(K \otimes A)^{+}\right)$, and also make the corresponding identification of the multiplier algebras. Thus $q$ becomes $\left(\begin{array}{ll}1 & 0 \\ 0 & 0\end{array}\right)$. Let

$$
\bar{\mu}: C\left(S^{1}\right) \otimes(K \otimes A)^{+} \rightarrow M_{2}\left(M\left(K\left(H^{2}\right) \otimes(K \otimes A)^{+}\right)\right)
$$

be the composition of $\mu$ with the identification just made.

Now define $\gamma: R K_{1}(S A) \rightarrow R K_{0}(A)$ as follows. Let $v \in U\left((K \otimes S A)^{+}\right)$ with $v-1 \in K \otimes S A$. View $(K \otimes S A)^{+}$as a subalgebra of $C\left(S^{1}\right) \otimes(K \otimes$ $A)^{+}$. Define $p=\bar{\mu}(v)^{*}\left(\begin{array}{ll}1 & 0 \\ 0 & 0\end{array}\right) \bar{\mu}(v)$. As we prove in Lemma 3.2 below, 
$p \in M_{2}\left(\left[K\left(H^{2}\right) \otimes K \otimes A\right]^{+}\right)$and $p-\left(\begin{array}{ll}1 & 0 \\ 0 & 0\end{array}\right) \in M_{2}\left(K\left(H^{2}\right) \otimes K \otimes A\right)$. Identify $K\left(H^{2}\right) \otimes K \otimes A$ with $K \otimes A$ via an isomorphism $K\left(H^{2}\right) \otimes K \rightarrow K$, and let $\gamma([v])$ be the resulting class of $p$ in $R K_{0}(A)$.

3.2 LeMmA. The maps $\beta$ and $\gamma$ of Definition 3.1 are well defined natural group homomorphisms.

Proof. Again, the only issue is well definedness. We do $\beta$ first. Using the notation of the definition, we first have to check that $u(\zeta)-1 \in M_{2}(K \otimes A)$ for $\zeta \in S^{1}$, and $u(1)=1$. The last is clear; for the first, a calculation shows that

$$
u(\zeta)-1=(\zeta-1) p\left(p-p_{0}\right)+(1-\bar{\zeta})\left(p-p_{0}\right) p_{0},
$$

which is in $M_{2}(K \otimes A)$ because $p-p_{0}$ is. It is now clear that a homotopy of projections yields a corresponding homotopy of unitaries, so that $\beta$ is in fact well defined.

We now turn to $\gamma$. We first have to check, in the notation of the definition, that $p \in M_{2}\left(\left[K\left(H^{2}\right) \otimes K \otimes A\right]^{+}\right)$and that $p-\left(\begin{array}{ll}1 & 0 \\ 0 & 0\end{array}\right) \in M_{2}\left(K\left(H^{2}\right) \otimes K \otimes A\right)$. The first relation will follow from the second, and the second is equivalent to $\mu(v)^{*} q \mu(v)-q \in K\left(L^{2}\right) \otimes K \otimes A$. Since $\mu(1)=1$, we get

$$
\mu(v)^{*} q \mu(v)-q=\mu(v)^{*}[q \mu(v-1)-\mu(v-1) q] .
$$

Now for $f \in C\left(S^{1}\right)$ it is a standard fact that $q_{0} \mu_{0}(f)-\mu_{0}(f) q_{0} \in K\left(L^{2}\right)$. (To prove this: a calculation shows that $q_{0} \mu_{0}\left(z^{n}\right)-\mu_{0}\left(z^{n}\right) q_{0}$ is an operator of rank $|n|$, so the general result follows by continuity.) It follows that $q \mu(a)-\mu(a) q \in K\left(L^{2}\right) \otimes K \otimes A$ for $a \in C\left(S^{1}\right) \otimes K \otimes A$. Taking $a=v-1$, we see that the right-hand side of $(*)$ is in $K\left(L^{2}\right) \otimes K \otimes A$, as desired.

Next, observe that $[p]$ does not depend on the choices of the isomorphisms $\left(H^{2}\right)^{\perp} \cong H^{2}$ or $K\left(H^{2}\right) \otimes K \cong K$, since in both cases, any two isomorphisms are homotopic. Finally, the construction of $p$ also converts a homotopy of unitaries into a homotopy of projections, showing that $\gamma([v])$ as defined depends only on $[v]$.

3.3 ReMARK. We will show in the next two lemmas that $\gamma=\beta^{-1}$. Since some of the calculations necessary in the $\sigma-C^{*}$-algebra case slightly obscure the essential simplicity of the proof, we describe the $C^{*}$-algebra case here.

Let $A$ be a unital $C^{*}$-algebra, and let $p \in A$ be a projection. Then the unitary in the definition of $\beta$ is the direct sum of a (large) identity and the unitary $u(\zeta)=1-p+\zeta p$ in $(S A)^{+}$. We have $\mu(u)=1 \otimes(1-p)+\mu_{0}(z) \otimes p$, where $\mu_{0}(z) \in L\left(L^{2}\right)$ is the bilateral shift (multiplication by $z$ ). Thus, 
$\mu(u)^{*} q \mu(u)=q+e \otimes p$, where $e \in L\left(L^{2}\right)$ is the rank one projection onto the space $\mathbb{C} \cdot z^{-1}$. Therefore $\gamma([u])=[e \otimes p]=[p]$, that is, $\gamma \circ \beta([p])=[p]$. It is easy enough to replace $A$ by $M_{n}(A)$, and to extend to classes of the form $\left[p_{1}\right]-\left[p_{2}\right]$ (it is convenient to take $p_{1} \perp p_{2}$ ), so as to conclude in the $C^{*}$-algebra case that $\gamma \circ \beta$ is the identity. (One uses the usual unitization method to deal with the nonunital case.)

To see the connection with Toeplitz operators, consider the Toeplitz operator $t=q \mu(u) q$ associated to $u$, for some unitary $u \in(S A)^{+}$. It is Fredholm in the appropriate sense because $u$ is invertible. If $u=1-p+z p$ as above, then $t=q_{0} \otimes(1-p)+s \otimes p$, where $s=q_{0} \mu_{0}(z) q_{0}$ is the unilateral shift. Thus, $t$ is a partial isometry with index $\left[1-t^{*} t\right]-\left[1-t t^{*}\right]=-[p]$. (See [18] for the appropriate notion of index.) It is easily shown in general that $\gamma([u])=-\operatorname{index}(t) \in K_{0}(A)$.

In the $C^{*}$-algebra case, the proof of Bott periodicity is now most easily completed by adapting Atiyah's trick from [1, Section 1]. The first thing to notice is that the internal products appearing in [1] are unnecessary, and that the external product $K_{0}(A) \times K_{0}(B) \rightarrow K_{0}\left(A \otimes_{\min } B\right)$ is easily defined by $([p],[q]) \mapsto[p \otimes q]$ for unital $A, B$, and extended to the nonunital case in the usual way. It follows that any natural map respects external products. One then proves that $\lambda_{\circ} \beta: K_{0}(A) \rightarrow K_{0}\left(S^{2} A\right)$ is given by the external product with the class $(\lambda \circ \beta)([1]) \in K_{0}\left(S^{2} \mathbb{C}\right)$, which is quite easy in the context of the usual definitions of $K$-theory for $\mathbb{C}^{*}$-algebras. Atiyah's argument now shows that the known relation $(\gamma \circ \nu) \circ(\lambda \circ \beta)=$ id for any $A$, combined with the nautrality of $\lambda \circ \beta$ and $\gamma \circ \nu$, implies that $(\lambda \circ \beta) \circ(\gamma \circ \nu)$ is also the identity.

In the $\sigma-C^{*}$-algebra case, the factor $\left(1-p_{0}+\zeta p_{0}\right)$ complicates the calculations, because we cannot require that $p$ commute with $p_{0}$. (The algebra $C(X)$ in [12, Example 4.9] is a counterexample.) The argument for $\gamma \circ \beta=\mathrm{id}$ is, however, essentially the same. In the argument for $\beta \circ \gamma=$ id, the formulas for external products are not so nice, and provide an additional complication. We find it simpler to give a direct proof. This proof is also a more useful model for a direct proof of the corresponding part of the main result of [14], mentioned in the introduction.

3.4 ReMARK. We should also say something about the connection between our proof and that of Cuntz in [5, Section 4]. Take his $E$ to be $K_{0}$, and interpret $E_{-1}$ as $K_{1}$, defined in terms of unitaries. Take the connecting homomorphism $\partial$ in the exact sequence $(*)$ of Section 2 to be as in [4, Definition 8.3.1]. We will describe the resulting isomorphism $\partial$ in [5, Theorem 4.4]. For simplicity, take $A$ to be unital, and consider only the classes of 
unitaries $u \in(S A)^{+}$. Then

$$
\partial([u])=\left[v\left(\begin{array}{ll}
1 & 0 \\
0 & 0
\end{array}\right) v^{*}\right]-\left[\left(\begin{array}{ll}
1 & 0 \\
0 & 0
\end{array}\right)\right] \in K_{0}\left((K \otimes A)^{+}\right),
$$

where $v \in M_{2}(T \otimes A)$ is a unitary whose image in $(S A)^{+}$is $u \oplus u^{*}$, and $T$, as in [5], is the Toeplitz algebra, that is, the $C^{*}$-algebra of the unilateral shift. With the identifications made in our definition of $\gamma$, we can take $v=\bar{\mu}(u)$. Thus $\partial=-\gamma$.

Cuntz proves directly that $E_{*}\left(T_{0}\right)=0$, where $T_{0}$ is a certain codimension one ideal in $T$. The proof is not as transparent as our approach, but it is short and actually proves a good deal more. In fact, Cuntz's proof applies essentially as it stands to functors on the category of $\sigma-C^{*}$-algebras satisfying his axioms (E1), (E2), (E3) ([5, Section 2]). (One needs Lemma 1.10 here.) In particular, his proof gives Bott periodicity immediately for the theory $R K_{*}$ as defined in [12]. But it is not clear how to generalize it to Fréchet algebras, while the proof given here does generalize ([16]).

3.5 LEMMA. The maps of Definition 3.1 satisfy $\gamma \circ \beta=\mathrm{id}$.

Proof. Let $p_{0}=\left(\begin{array}{ll}1 & 0 \\ 0 & 0\end{array}\right)$ as usual, and let $p \in M_{2}\left((K \otimes A)^{+}\right)$be a projection with $p-p_{0} \in M_{2}(K \otimes A)$. Let $u=(1-p+z p)\left(1-p_{0}+z p_{0}\right)^{*}$ be the unitary representing $\beta([p])$ as in Definition 3.1; we postpone the application of the isomorphism $M_{2}(K) \rightarrow K$ used there until the end of the proof.

We now compute $\mu(u)^{*} q \mu(u)$. For this we need some notation. Let $e_{i j} \in$ $L\left(L^{2}\right)$ be the rank one partial isometry sending $z^{j}$ to $z^{i}$ and vanishing on $\left(\mathbb{C} z^{j}\right)^{\perp}$, and abbreviate $e_{i i}$ to $e_{i}$. Then

$$
\begin{aligned}
\mu(u)^{*} q \mu(u)= & \\
& {\left[1 \otimes\left(1-p_{0}\right)+\mu(z)\left(1 \otimes p_{0}\right)\right] \cdot\left[1 \otimes(1-p)+\mu(z)^{*}(1 \otimes p)\right] \cdot q } \\
& \cdot[1 \otimes(1-p)+\mu(z)(1 \otimes p)] \cdot\left[1 \otimes\left(1-p_{0}\right)+\mu(z)^{*}\left(1 \otimes p_{0}\right)\right] \\
= & {\left[1 \otimes\left(1-p_{0}\right)+\mu(z)\left(1 \otimes p_{0}\right)\right] } \\
& \cdot\left[q+e_{-1} \otimes p\right] \cdot\left[1 \otimes\left(1-p_{0}\right)+\mu(z)^{*}\left(1 \otimes p_{0}\right)\right] \\
= & q-e_{0} \otimes p_{0}+e_{-1} \otimes\left(1-p_{0}\right) p\left(1-p_{0}\right)+e_{-1,0} \otimes\left(1-p_{0}\right) p p_{0} \\
& +e_{0,-1} \otimes p_{0} p\left(1-p_{0}\right)+e_{0} \otimes p_{0} p p_{0} .
\end{aligned}
$$

The first step is the application of $\mu$ to $u$, the second is obtained by multiplying together the middle three terms, and the third by multiplying out and identifying $\mu(z)$ as the bilateral shift. Now choose an isomorphism of $\left(H^{2}\right)^{\perp}$ with $H^{2}$; we take the one sending $z^{-i}$ to $z^{i-1}$ for $i \geq 1$. Thus, the isomorphism $K\left(L^{2}\right) \rightarrow M_{2}\left(K\left(H^{2}\right)\right)$ sends $e_{0}$ to $\left(\begin{array}{ll}e_{0} & 0 \\ 0 & 0\end{array}\right), e_{-1}$ to $\left(\begin{array}{ll}0 & 0 \\ 0 & e_{0}\end{array}\right), e_{0,-1}$ to 
$\left(\begin{array}{cc}0 & e_{0} \\ 0 & 0\end{array}\right)$, and $e_{-1,0}$ to $\left(\begin{array}{ll}0 & 0 \\ e_{0} & 0\end{array}\right)$. We further remember that $p_{0}=\left(\begin{array}{ll}1 & 0 \\ 0 & 0\end{array}\right)$, and we

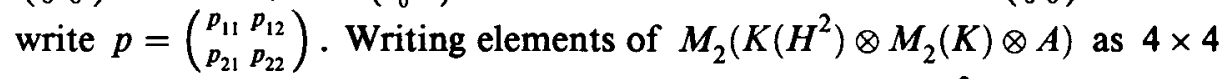
matrices, with $2 \times 2$ blocks representing elements of $K\left(H^{2}\right) \otimes M_{2}(K) \otimes A$, we get

$$
\mu(u)^{*} q \mu(u)=\left[\begin{array}{ccc|cc}
\left(1-e_{0}\right) \otimes 1+e_{0} \otimes p_{11} & 0 & 0 & e_{0} \otimes p_{12} \\
0 & 1 & 0 & 0 \\
\hline 0 & 0 & 0 & 0 \\
e_{0} \otimes p_{21} & 0 & 0 & e_{0} \otimes p_{22}
\end{array}\right] .
$$

This projection is homotopic within the appropriate class to

$$
\begin{aligned}
& f=\left[\begin{array}{cc|cc}
\left(1-e_{0}\right) \otimes 1+e_{0} \otimes p_{11} & 0 & e_{0} \otimes p_{12} & 0 \\
0 & 1 & 0 & 0 \\
\hline e_{0} \otimes p_{21} & 0 & e_{0} \otimes p_{22} & 0 \\
0 & 0 & 0 & 0
\end{array}\right] \\
& =\left(1-e_{0}\right) \otimes\left[\begin{array}{llll}
1 & & & 0 \\
& 1 & & \\
& & 0 & \\
0 & & & 0
\end{array}\right]+e_{0} \otimes\left[\begin{array}{ccc|cc}
p_{11} & 0 & p_{12} & 0 \\
0 & 1 & 0 & 1 \\
\hline p_{21} & 0 & p_{21} & 0 \\
0 & 0 & 0 & 0
\end{array}\right] \text {. }
\end{aligned}
$$

Let $H$ be the space on which $K$ acts, and set $H_{1}=e_{0} H^{2} \otimes H$ and $H_{2}=$ $\left[\left(1-e_{0}\right) H^{2} \otimes H\right] \oplus\left[H^{2} \otimes H\right]$. Then $f$ is recognizable as the direct sum of a copy of $p$ in $M_{2}\left(\left(K\left(H_{1}\right) \otimes A\right)^{+}\right)$and a copy of $\left(\begin{array}{ll}1 & 0 \\ 0 & 0\end{array}\right)$ in $M_{2}\left(\left(K\left(H_{2}\right) \otimes A\right)^{+}\right)$. Since $H_{1} \cong H_{2} \cong H$ and $\left(\begin{array}{ll}1 & 0 \\ 0 & 0\end{array}\right)$ is the identity of $R K_{0}(A)$, we have proved that $\left[\mu(u)^{*} q \mu(u)\right]=[p]$, that is, that $\gamma \circ \beta([p])=[p]$.

3.6 LEMMA. The maps of Definition 3.1 satisfy $\beta \circ \gamma=\mathrm{id}$.

Proof. Let $v \in(K \otimes S A)^{+}$be a unitary with $v-1 \in K \otimes S A$. Then $\beta \circ \gamma([v])$ is the class of the unitary

$$
\zeta \mapsto u(\zeta)=\left[1-\mu(v)^{*} q \mu(v)+\zeta \mu(v)^{*} q \mu(v)\right] \cdot[1-q+\zeta q]^{*} .
$$

We may disregard the identification $K\left(L^{2}\right) \cong M_{2}\left(K\left(H^{2}\right)\right) \cong K$, and we therefore regard $u$ as an element of $B=C\left(S^{1}\right) \otimes M\left(K\left(L^{2}\right) \otimes(K \otimes A)^{+}\right)$. We rewrite it as

$$
\begin{aligned}
u & =[1 \otimes \mu(v)]^{*} \cdot[1-1 \otimes q+z \otimes q] \cdot[1 \otimes \mu(v)] \cdot[1-1 \otimes q+z \otimes q]^{*} \\
& =\eta_{0}(v)^{*} \psi_{0}(v),
\end{aligned}
$$

where $\eta_{0}, \psi_{0}: C\left(S^{1}\right) \otimes(K \otimes A)^{+} \rightarrow B$ are given by $\eta_{0}(b)=1 \otimes \mu(b) \quad$ and $\quad \psi_{0}(b)=[1-1 \otimes q+z \otimes q] \eta_{0}(b)[1-1 \otimes q+z \otimes q]^{*}$. 
We now want to construct certain homotopies of homomorphisms from $C\left(S^{1}\right) \otimes(K \otimes A)^{+}$to $B$. It follows from [11, Proposition 3.3] (which is standard for $C^{*}$-algebras) and the fact that $C\left(S^{1}\right)$ is the univeral $C^{*}$-algebra generated by a unitary (namely $z$ ) that unital homomorphisms $\varphi: C\left(S^{1}\right) \otimes$ $(K \otimes A)^{+} \rightarrow B$ are in one to one correspondence with pairs consisting of a unitary $w \in B$ and a unital homomorphism $\bar{\varphi}:(K \otimes A)^{+} \rightarrow B$ such that $w$ commutes with all $\bar{\varphi}(a)$. (The tensor product in [10, Proposition 3.3] is the maximal tensor product, but, since $C\left(S^{1}\right)$ is nuclear, all tensor products are the same.) The relation between $\varphi, w$, and $\bar{\varphi}$ is of course $w=\varphi(z \otimes 1)$ and $\bar{\varphi}(a)=\varphi(1 \otimes a)$.

We will write elements of $B=C\left(S^{1}\right) \otimes M\left(K\left(L^{2}\right) \otimes(K \otimes A)^{+}\right)$as infinite matrices relative to the basis of $L^{2}$ consisting of the power of $z$, with lines between the -1 and 0 columns and between the -1 and 0 rows to indicate the indexing. The entries are of course elements of $C\left(S^{1}\right) \otimes(K \otimes A)^{+}$. The homomorphism $\bar{\eta}_{0}$ obtained from the previous paragraph is just $a \mapsto$ $\eta_{0}(1 \otimes a)$, and the unitary is the bilateral shift

$$
\eta_{0}(z \otimes 1)=1 \otimes \mu(z \otimes 1)=\left[\begin{array}{ccc|cccc}
\ddots & & & & & & 0 \\
1 & 0 & & & & \\
& 1 & 0 & & & \\
\hline & & 1 & 0 & & \\
& & & 1 & 0 & \\
0 & & & & 1 & \ddots
\end{array}\right] .
$$

One checks readily that $\bar{\psi}_{0}=\bar{\eta}_{0}$, and that the corresponding unitary is

$$
\psi_{0}(z \otimes 1)=\left[\begin{array}{ccc|ccc}
\ddots & & & & & \\
1 & 0 & & & & \\
& 1 & 0 & & & \\
\hline & & z & 0 & & \\
& & & 1 & 0 & \\
0 & & & & 1 & \ddots
\end{array}\right] .
$$

Define a unitary $c_{t}$ for $t \in[0,1]$ by

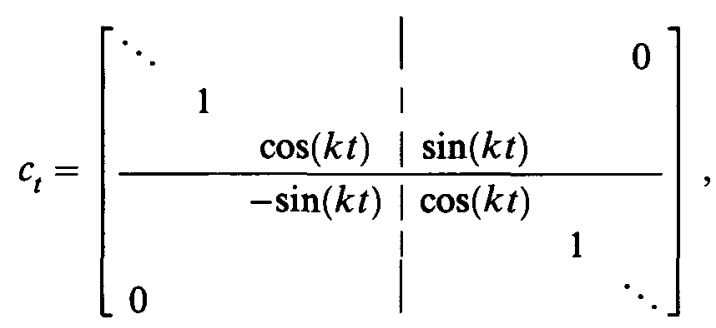


with $k=\pi / 2$. Clearly $c_{t}$ commutes with all $\mu(1 \otimes a)$, so we can define homomorphisms $\eta_{t}$ and $\psi_{t}$ by $\eta_{t}(z \otimes 1)=\eta_{0}(z \otimes 1) c_{t}$ and $\eta_{t}(1 \otimes a)=$ $\eta_{0}(1 \otimes a)$, and by $\psi_{t}(z \otimes 1)=\psi_{0}(z \otimes 1) c_{t}$ and $\psi_{t}(1 \otimes a)=\eta_{0}(1 \otimes a)$. These agree with the earlier definitions of $\eta_{0}$ and $\psi_{0}$ because $c_{0}=1$.

We now verify an important property, namely that

$(*) \eta_{t}(b)-\psi_{t}(b) \in C_{0}\left(S^{1}-\{1\}\right) \otimes K\left(L^{2}\right) \otimes(K \otimes A) \quad$ for $b \in C\left(S^{1}\right) \otimes(K \otimes A)$.

First observe that

$$
\eta_{t}(z \otimes 1)-\psi_{t}(z \otimes 1)=\left[\eta_{0}(z \otimes 1)-\psi_{0}(z \otimes 1)\right] c_{t}
$$

The first factor is in the ideal $I=C_{0}\left(S^{1}-\{0\}\right) \otimes K\left(L^{2}\right) \otimes(K \otimes A)^{+}$, whence so is the product. It follows that $\eta_{t}\left(z^{n} \otimes 1\right)-\psi_{t}\left(z^{n} \otimes 1\right) \in I$ for all $n$. Furthermore, for $a \in K \otimes A$ we have

$$
\eta_{t}(1 \otimes a)=\psi_{t}(1 \otimes a)=\eta_{0}(1 \otimes a) \in \mathbb{C} \cdot 1 \otimes \mathbb{C} \cdot 1 \otimes(K \otimes A) .
$$

Therefore

$$
\begin{aligned}
\eta_{t}\left(z^{n} \otimes a\right)-\psi_{t}\left(z^{n} \otimes a\right) & =\left[\eta_{t}\left(z^{n} \otimes 1\right)-\psi_{t}\left(z^{n} \otimes 1\right)\right] \eta_{0}(1 \otimes a) \\
& \in C_{0}\left(S^{1}-\{0\}\right) \otimes K\left(L^{2}\right) \otimes(K \otimes A),
\end{aligned}
$$

as desired. As a consequence, we obtain

$\eta_{t}(v)^{*} \psi_{t}(v)-1=\eta_{t}(v)^{*}\left[\psi_{t}(v-1)-\eta_{t}(v-1)\right] \in C_{0}\left(S^{1}-\{0\}\right) \otimes K\left(L^{2}\right) \otimes(K \otimes A)$,

that is, $\eta_{t}(v)^{*} \psi_{t}(v) \in\left(K\left(L^{2}\right) \otimes K \otimes S A\right)^{+}$with $\eta_{t}(v)^{*} \psi_{t}(v)-1 \in K\left(L^{2}\right) \otimes$ $K \otimes S A$. We have thus constructed a homotopy from $\eta_{0}(v)^{*} \psi_{0}(v)=u$ to $\eta_{1}(v)^{*} \psi_{1}(v)$.

Now compute

$$
\eta_{1}(z \otimes 1)=\eta_{0}(z \otimes 1) c_{1}=\left[\begin{array}{cccc|ccccc}
\ddots & & & & & & & & 0 \\
& 1 & 0 & & & & & & \\
& 1 & 0 & & & & \\
\hline & & 0 & 1 & & & \\
& & & -1 & 0 & 0 & & \\
& & & & & 1 & 0 & \\
0 & & & & & & & 1 & \ddots
\end{array}\right]
$$


and

$$
\psi_{1}(z \otimes 1)=\psi_{0}(z \otimes 1) c_{1}=\left[\begin{array}{cccc|cccc}
\ddots & & & & & & & \\
& 1 & 0 & & \mid & & & \\
& & 1 & 0 & \mid & & & \\
\hline & & 0 & z & & & \\
& & -1 & 0 & 0 & & \\
& & & & & 1 & 0 & \\
0 & & & & & & 1 & \ddots
\end{array}\right] .
$$

Let $e_{0}$ be the projection on the constant functions in $L^{2}$, and let $\bar{e}_{0}=$ $1 \otimes e_{0} \otimes 1$. Then $\bar{e}_{0}$ commutes with $\eta_{1}(z \otimes 1)$ and $\psi_{1}(z \otimes 1)$, also with all $\eta_{0}(1 \otimes a)$, and hence with all $\eta_{1}(b)$ and $\psi_{1}(b)$. Furthermore, (1$\left.\bar{e}_{0}\right) \psi_{1}(b)\left(1-\bar{e}_{0}\right)=\left(1-\bar{e}_{0}\right) \eta_{1}(b)\left(1-\bar{e}_{0}\right)$, while $\bar{e}_{0} \eta_{1}(f \otimes a) \bar{e}_{0}=f(1) \otimes e_{0} \otimes a$ and $\bar{e}_{0} \psi_{1}(f \otimes a) \bar{e}_{0}=f \otimes e_{0} \otimes a$. (One checks these formulas on $z \otimes 1$ and on $1 \otimes a$.) Therefore

$$
\eta_{1}(v)^{*} \psi_{1}(v)=\left[\begin{array}{ccc|cccc}
\ddots & & & & & 0 & \\
& 1 & & & & \\
& & 1 & & & \\
& & & v & & & \\
& & & & & 1 & \\
& & & & & & \\
0 & & & & & & \ddots
\end{array}\right]
$$

which we can regard as $v \oplus 1$. Thus,

$$
\gamma \circ \beta([v])=\left[\eta_{0}(v)^{*} \psi_{0}(v)\right]=\left[\eta_{1}(v)^{*} \psi_{1}(v)\right]=[v \oplus 1]=[v],
$$

as desired.

3.7 Theorem. There is a natural isomorphism $R K_{1}(S A) \cong R K_{0}(A)$.

Proof. According to the previous two lemmas, we have $\gamma \circ \beta=$ id and $\beta \circ \gamma=\mathrm{id}$.

\section{A Simplified Construction of Representable $K$-Theory}

In [12], we defined $R K_{0}(A)$, for $A$ unital, to be the set of homotopy classes in the unitary group of the stable outer multiplier algebra $M(K \otimes A) /(K \otimes A)$. This definition was motivated by the definition of representable $K$-theory of a space as the set of homotopy classes of maps to the Fredholm operators 
(see for example [7]), and by Mingo's work [9], which shows that this definition gives the correct answer for $C^{*}$-algebras. The definition was extended to nonunital $\sigma-C^{*}$-algebras by considering the unitization, and to $R K_{1}$ by suspending. The proofs of the basic properties of the theory required some messy lemmas involving multiplier algebras. (See [12, Section 1]). The proof of the Milnor $\lim ^{1}$-sequence was messy, and the proof of Bott periodicity was obtained by reduction to the $C^{*}$-algebra case. Furthermore, in the construction of equivariant representable $K$-theory, done in [12, Section 5], additional messy lemmas were needed and the group was assumed to be second countable.

In this section, we show how to develop the basic properties of representable $K$-theory from Definition 1.1. This development is much cleaner and more straightforward, and much more closely parallels the usual presentation of $K$-theory for Banach algebras or $C^{*}$-algebras in, for example, [19] and [4, Sections 5, 8, and 9]. This method also generalizes cleanly to the equivariant case, for an arbitrary compact group. Most of the work has already been done, either here or elsewhere. All that remains is to prove one lemma, slightly modify several proofs, and put the pieces together in the proper order.

4.1 LeMMA. Let $0 \rightarrow I \stackrel{\leftrightarrow}{\rightarrow} A \stackrel{\pi}{\rightarrow} B \rightarrow 0$ be an exact sequence of $\sigma \cdot C^{*}$. algebras. Then there is a natural exact sequence

$$
\begin{aligned}
\cdots \rightarrow R K_{0}\left(S^{2} A\right) \stackrel{\pi_{*}}{\rightarrow} R K_{0}\left(S^{2} B\right) & \rightarrow R K_{0}(S I) \stackrel{\leftrightarrow}{\rightarrow} R K_{0}(S A) \stackrel{\pi_{*}}{\rightarrow} R K_{0}(S B) \\
& \rightarrow R K_{0}(I) \stackrel{\iota^{*}}{\rightarrow} R K_{0}(A) \stackrel{\pi_{*}}{\rightarrow} R K_{0}(B) .
\end{aligned}
$$

Proof. We only have to prove exactness at $R K_{0}(A)$; the rest follows from the homotopy invariance of $R K_{0}$ by a standard argument, given, for example, in [8, Lemma 5 of Section 7]. (Also see [12, Corollary 2.5].) It is also trivial to check that $\pi_{*} \circ l_{*}=0$. So we prove $\operatorname{Ker}\left(\pi_{*}\right) \subset \operatorname{Im}\left(l_{*}\right)$.

Let $p \in M_{2}\left((K \otimes A)^{+}\right)$be a projection satisfying $p-\left(\begin{array}{ll}1 & 0 \\ 0 & 0\end{array}\right) \in M_{2}(K \otimes A)$ and $\pi_{*}([p])=0$. Then there is a homotopy $t \mapsto q_{t}$ from $q_{0}=\pi(p)$ to $q_{1}=\left(\begin{array}{ll}1 & 0 \\ 0 & 0\end{array}\right)$ in $M_{2}\left((K \otimes B)^{+}\right)$such that $q_{t}-\left(\begin{array}{ll}1 & 0 \\ 0 & 0\end{array}\right) \in M_{2}(K \otimes B)$. By Lemma 1.9 , there is a unitary path $t \mapsto v_{t}$ in $M_{2}\left((K \otimes B)^{+}\right)$with $v_{1}=1, v_{t} q_{t} v_{t}^{*}=$ $\left(\begin{array}{ll}1 & 0 \\ 0 & 0\end{array}\right)$, and $v_{t}-1 \in M_{2}(K \otimes B)$. Now use Lemma 1.8 to lift $v$ to a unitary path $t \mapsto u_{t}$ in $M_{2}\left((K \otimes A)^{+}\right)$with $u_{1}=1$ and $u_{t}-1 \in M_{2}(K \otimes A)$. The homotopy $t \mapsto u_{t} p u_{t}^{*}$ shows that $[p]=\left[u_{0} p u_{0}^{*}\right]$. Furthermore, $\pi\left(u_{0} p u_{0}^{*}\right)=$ $v_{0} q_{0} v_{0}^{*}=\left(\begin{array}{ll}1 & 0 \\ 0 & 0\end{array}\right)$, so $u_{0} p u_{0}^{*}-\left(\begin{array}{ll}1 & 0 \\ 0 & 0\end{array}\right) \in M_{2}(K \otimes I)$. Thus, $\left[u_{0} p u_{0}^{*}\right]$ is an element of $R K_{0}(I)$ with $u_{*}\left(\left[u_{0} p u_{0}^{*}\right]\right)=[p]$. 
4.2 Outline of the development of representable $K$-theory for $\sigma$ - $C^{*}$-algebras. Step 1. Prove the two basic lemmas on projections and unitaries stated here as Lemmas 1.8 and 1.9. Note that the profs of these lemmas are reasonably simple, and do not depend on other material in the papers from which they are taken.

Step 2. Define $R K_{0}(A)$ and $R K_{1}(A)$ for a $\sigma-C^{*}$-algebra $A$, and prove that they are homotopy invariant functorial abelian groups, and agree with the usual $K$-groups on $C^{*}$-algebras, as was done here in Section 1.

Step 3. Prove Lemma 4.1, the one-sided long exact sequence for $R K_{0}$.

Step 4. Prove Bott periodicity, $R K_{i}(S A) \cong R K_{1-i}(A)$, as in Sections 2 and 3. The part of the exact sequence shown in $(*)$ of Lemma 4.1 is then just enough to give the usual six term exact sequence

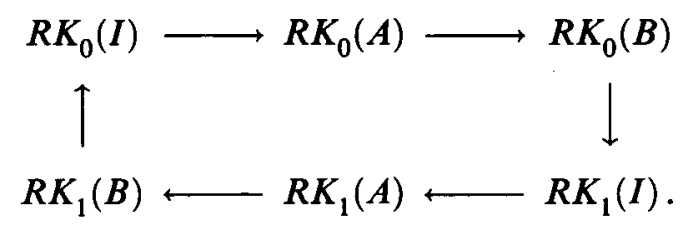

Step 5. Prove the Mayer-Vietoris sequence: if $A$ is a $\sigma-C^{*}$-algebra, and $J_{0}$ and $J_{1}$ are closed ideals in $A$, then there is a natural six term exact sequence

$$
\begin{gathered}
R K_{0}\left(A /\left(J_{0} \cap J_{1}\right)\right) \rightarrow R K_{0}\left(A / J_{0}\right) \oplus R K_{0}\left(A / J_{1}\right) \rightarrow R K_{0}\left(A /\left(J_{0}+J_{1}\right)\right) \\
\uparrow
\end{gathered}
$$

with the usual formulas for the maps. (See [12, Proposition 2.8].) Since we already have the six term exact sequence $(* *)$, the proof of $[17$, Theorem $4.1]$ is directly applicable. Alternatively, the proof used for [12, Proposition 2.8] now gives the entire sequence without truncation, and [12, Lemma 2.6] becomes trivial.

Step 6. Prove countable additivity: $R K_{i}\left(\prod_{n} A_{n}\right) \cong \prod_{n} R K_{i}\left(A_{n}\right)$, for $\sigma-$ $C^{*}$-algebras $A_{n}[12$, Proposition 3.1]. The proof is even simpler than the proof given there.

Step 7. Prove the Milnor $\lim ^{1}$-sequence ([12, Theorem 3.2]). The proof is simpler than there. With $\overleftarrow{A=} \lim A_{n}$, and other notation as in [12], the first part of that proof gives the exact sequence

$$
0 \rightarrow \lim ^{1} R K_{1-i}\left(A_{n}\right) \rightarrow R K_{i}(T) \rightarrow \lim _{\longleftarrow} R K_{i}\left(A_{n}\right) \rightarrow 0
$$

for $i=0$ and $i=1$, and the next part gives $R K_{i}(T) \cong R K_{i}(A)$, again for both $i=0$ and $i=1$. The rest of the proof in [12], consisting of special 
arguments for the case $i=0$, is now superfluous. In particular, nothing resembling the proof of $[12$, Lemma 2.6] is ever needed.

Step 8. Prove stability: $R K_{i}(K(H) \otimes A) \cong R K_{i}(A)$ for any Hilbert space $H$. This is essentially immediate from the definition if $H$ is finite-dimensional or separable infinite dimensional. For nonseparable $H$, an attempt to use the usual sort of approximation argument seems to require methods similar to those of [13]. The simplest thing to do is to choose a rank one projection $e \in K(H)$, observe that $a \mapsto e \otimes a$ defines an isomorphism on the $K$-theory of $C^{*}$-algebras, and use the Milnor $\lim ^{1}$-sequence to conclude that this is also true for $\sigma-C^{*}$-algebras.

We now consider equivariant $K$-theory. For the rest of this section, we let $G$ be an arbitrary, but fixed, compact group. We assume that $G$ acts continuously on all $\sigma-C^{*}$-algebras ([12, Definition 5.1]), and that all homomorphisms are equivariant. We use $[12$, Lemma 5.2] without comment. The superscript $G$ indicates the fixed point algebra. $G$ always acts by conjugation by the regular representation on $K\left(L^{2}(G)\right)$, trivially on $K, C\left(S^{1}\right)$, etc., and via the diagonal action on tensor products. For equivariant $K$-theory of $C^{*}$-algebras, we refer to [10].

4.3 Definition. Let $A$ be a $\sigma-C^{*}$-algebra with a continuous action of $G$. Then $R K_{0}^{G}(A)$ is the set of homotopy classes of $G$-invariant projections $p \in M_{2}\left(\left[K \otimes K\left(L^{2}(G)\right) \otimes A\right]^{+}\right)$such that $p-\left(\begin{array}{ll}1 & 0 \\ 0 & 0\end{array}\right) \in M_{2}\left(K \otimes K\left(L^{2}(G)\right) \otimes\right.$ $A$ ). Also, $R K_{1}^{G}(A)$ is the set of homotopy classes in the set of $G$-invariant unitaries $u \in\left[K \otimes K\left(L^{2}(G)\right) \otimes A\right]^{+}$such that $u-1 \in K \otimes K\left(L^{2}(G)\right) \otimes A$. Additoin and induced maps are defined as for $R K_{0}(A)$ and $R K_{1}(A)$, using the isomorphism $M_{2}(K) \cong K$ exactly as before. We further let $R(G)$ be the representation ring of $G$ (see [18]), and define an $R(G)$-module structure on $R K_{i}^{G}(A)$ as follows. If $V$ is a finite dimensional unitary representation space of $G$, then $V \otimes L^{2}(G)$ is isomorphic to the direct sum of $\operatorname{dim}(V)$ copies of $L^{2}(G)$, so that $K \otimes K\left(L^{2}(G)\right) \cong K \otimes K\left(L^{2}(G)\right) \otimes K(V)$. Define $[V][p]=\left[1_{V} \otimes p\right]$, where $1_{V}$ is the identity operator on $V$, and $1_{V} \otimes p$ defines a class in $R K_{0}^{G}(A)$ by the isomorphism just obtained. Similarly define $[V][u]=\left[1_{V} \otimes u\right]$ in $R K_{1}^{G}(A)$.

4.4 THEOREM. (Compare [12, Theorem 5.8].) $R K_{*}^{G}$ is a functor from $\sigma$ $C^{*}$-algebras to $R(G)$-module satisfying the following properties.

(1) Homotopy invariance: if $\varphi$ and $\psi$ are G-homotopic homomorphisms, then $\varphi_{*}=\psi_{*}$.

(2) Long exact sequence. if $0 \rightarrow I \rightarrow A \rightarrow B \rightarrow 0$ is an equivariant short exact sequence of $\sigma-C^{*}$-algebras, then there is a natural six term exact 
sequence

$$
\begin{gathered}
R K_{0}^{G}(I) \longrightarrow R K_{0}^{G}(A) \longrightarrow R K_{0}^{G}(B) \\
\uparrow \\
R K_{1}^{G}(B) \longleftarrow R K_{1}^{G}(A) \longleftrightarrow R K_{1}^{G}(I) .
\end{gathered}
$$

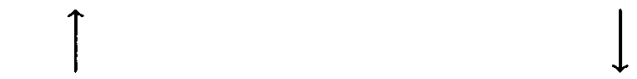

(3) Bott periodicity: there are natural isomorphisms $R K_{i}^{G}(S A) \cong R K_{i-1}^{G}(A)$ for every $A$.

(4) More Bott periodicity: if $V$ is a finite dimensional complex vector space on which $G$ acts via a unitary representation, then there is a natural isomorphism $R K_{i}^{G}\left(C_{0}(V) \otimes A\right) \cong R K_{i}^{G}(A)$ for every $A$.

(5) Stability: if $V$ is any Hilbert space on which $G$ acts via a unitary representation, then there is a natural isomorphism $R K_{i}^{G}(K(V) \otimes A) \cong R K_{i}^{G}(A)$ for every $A$.

(6) Milnor $\varliminf^{1}$-sequence: if $A=\lim _{n} A_{n}$ equivariantly, then there is a natural short exact sequence

$$
0 \rightarrow \lim ^{1} R K_{1-i}^{G}\left(A_{n}\right) \rightarrow R K_{i}^{G}(A) \rightarrow \underset{\lim }{\longleftarrow} R K_{i}^{G}\left(A_{n}\right) \rightarrow 0 .
$$

(7) Green-Julg-Rosenberg Theorem: there are natural isomorphisms $R K_{i}^{G}(A) \cong R K_{i}\left(C^{*}(G, A)\right)$ for every $A$.

(8) Agreement with $K$-theory: if $A$ is a $C^{*}$-algebra, then there are natural isomorphisms $R K_{i}^{G}(A) \cong K_{i}^{G}(A)$.

(9) Exterior invariance: if two actions of $G$ on $A$ are exterior equivalent ([10, Definition 2.7.1]), then $R K_{i}^{G}(A)$ is the same for both actions.

Proof. We immediately see that, as a group, $R K_{i}^{G}(A)$ has been essentially defined to be $R K_{i}\left(\left[K\left(L^{2}(G)\right) \otimes A\right]^{G}\right)$. Thus, what we already know about $R K_{i}$ gives us the fact that each $R K_{i}^{G}(A)$ is a functorial abelian group and parts (1), (2), (3), and (6). We also get (5) this way, because if $V$ is any Hilbert space on which $G$ acts via a unitary representation, then $V \otimes L^{2}(G)$ is isomorphic to a Hilbert direct sum of $\operatorname{dim}(V)$ copies of $L^{2}(G)$. For part (8), an examination of the definition of $K_{0}^{G}(A)$ in terms of projections for a unital $C^{*}$-algebra $A$ ([10, Section 2.4]) shows that is also has been essentially defined to be $K_{0}\left(\left[K\left(L^{2}(G)\right) \otimes A\right]^{G}\right)$. (Note that $L^{2}(G)$ contains every irreducible representation of $G$.) This gives (8) for unital algebras and $i=0$, and the rest follows by taking unitizations and suspensions.

Next observe that the multiplications by $[V] \in R(G)$ are just the induced maps on representable $K$-theory coming from $a \mapsto 1_{V} \otimes a$, regarded as a homomorphism from $\left[K\left(L^{2}(G)\right) \otimes A\right]^{G}$ to $\left[K(V) \otimes K\left(L^{2}(G)\right) \otimes A\right]^{G}$. We 
omit the routine verification that these do in fact define an $R(G)$-module structure on $R K_{i}^{G}(A)$. Naturality in parts (1), (2), (3), (5), and (6) now ensures that the maps there are also all $R(G)$-module homomorphisms, and comparison with [10, Chapter 2] does the same for (8).

For (7), we observe that noncommutative duality theory, as summarized in [6, Section 1], implies that $\left[K\left(L^{2}(G)\right) \otimes A\right]^{G}$ is naturally isomorphic to $C^{*}(G, A)$ if $A$ is a $C^{*}$-algebra, and therefore also (by taking inverse limits) if $A$ is a $\sigma-C^{*}$-algebra. With the $R(G)$-module structure on $R K_{i}\left(C^{*}(G, A)\right)$ defined as in [10, Section 2.7], the resulting isomorphism $R K_{i}^{G}(A) \cong$ $R K_{i}\left(C^{*}(G, A)\right)$ is an $R(G)$-module homomorphism for $C^{*}$-algebras, and hence also for $\sigma-C^{*}$-algebras.

We now get (9) by observing that crossed products by exterior equivalent actions are naturally isomorphic when $A$ is a $C^{*}$-algebra, and thus when $A$ is a $\sigma-C^{*}$-algebra. Only (4) remains; to prove that, recall that the isomorphism $\beta: K_{i}^{G}(A) \rightarrow K_{i}^{G}\left(C_{0}(V) \otimes A\right)$ is given by the external product with a class $\eta \in K_{0}\left(C_{0}(V)\right)$. Write $\eta=[p]-[q]$ with $p$ and $q$ projections in some $K(W) \otimes C_{0}(V)^{+}$, and let $\varphi, \psi: A \rightarrow K(W) \otimes C_{0}(V)^{+} \otimes A$ be $\varphi(a)=p \otimes a$ and $\psi(a)=q \otimes a$. Note that the images of $p$ and $q$ in $K(W)$ are equivalent and hence $G$-homotopic. Therefore, if $\pi: K(W) \otimes C_{0}(V)^{+} \otimes A \rightarrow K(W) \otimes A$ is the quotient map associated with the unitization $C_{0}(V)^{+}$, we have $\pi_{*} \circ \varphi_{*}=\pi_{*} \circ$ $\psi_{*}$, so that $\varphi_{*}-\psi_{*}$ defines a map $R K_{i}^{G}(A) \rightarrow R K_{i}^{G}\left(K(W) \otimes C_{0}(V) \otimes A\right)$. This map is natural, and (using stability) is the isomorphism $\beta$ when $A$ is a $C^{*}$. algebra, and thus by the Milnor $\lim ^{1}$-sequence, $\varphi_{*}-\psi_{*}$ is an isomorphism for any $\sigma-C^{*}$-algebra $A$.

4.5 REMARK. We never used the original Julg's Theorem (see [10, Section 2.6]) in our proof of the corresponding part (7) of the previous theorem. In fact, our methods give an immediate proof of Julg's theorem for $C^{*}$ algebras. One notices, for unital $A$, that $K_{0}^{G}(A)$ is essentially defined to be $K_{0}\left(\left[K\left(L^{2}(G)\right) \otimes A\right]^{G}\right)$. (This is best seen in [10, Section 2.4].) Noncommutative duality theory, as in $[6$, Section 1$]$, gives $C^{*}\left(G, C^{*}(G, A, \alpha), \delta\right) \cong$ $K\left(L^{2}(G)\right) \otimes A$ equivariantly, where $\alpha$ is the action of $G$ on $A$ and $\delta$ is the dual coaction. It is trivial to check that for any coaction $\delta$ of a compact group $G$ on a $C^{*}$-algebra $B$, one has $C^{*}(G, B, \delta)^{G} \cong B$. So $\left[K\left(L^{2}(G)\right) \otimes A\right]^{G} \cong C^{*}(G, A)$, and $K_{0}^{G}(A) \cong K_{0}\left(C^{*}(G, A)\right)$. 


\section{References}

[1] M. F. Atiyah, 'Bott periodicity and the index of elliptic operators', Quart. J. Math. Oxford Ser. 2, 19 (1968), 113-140.

[2] M. F. Atiyah, 'Algebraic topology and operators in Hilbert space', in: Lectures in modern analysis I (ed. C. T. Taam), Lecture Notes in Math. 103 (Springer, Berlin, 1969), pp. 101-121.

[3] M. F. Atiyah and R. Bott, 'On the periodicity theorem for complex vector bundles', Acta Math. 112 (1964), 229-247.

[4] B. Blackadar, $K$-theory for operator algebras, MSRI publications no. 5 (Springer-Verlag, Berlin, 1986).

[5] J. Cuntz, ' $K$-theory and $C^{*}$-algebras', in: Algebraic $K$-theory, number theory, geometry, and analysis, Proceedings, Bielefeld 1982 (ed. A. Bak), Lecture Notes in Math. 1046, (Springer, Berlin, 1984), pp. 55-79.

[6] E. C. Gootman and A. J. Lazar, 'Applications of non-commutative duality to crossed product $C^{*}$-algebras determined by an action or coaction', Proc. London Math. Soc. 59 (1989), 593-624.

[7] M. Karoubi, 'Espaces classifiants en K-théorie', Trans. Amer. Math. Soc. 147 (1970), 75-115.

[8] G. G. Kasparov, 'The operator $K$-functor and extensions of $C^{*}$-algebras', Izv. $A k a d$. Nauk SSSR, Ser. Mat. 44 (1980), 571-636 (in Russian); English translation in Math. USSR Izvestija 16 (1981), 513-572.

[9] J. A. Mingo, ' $K$-theory and multipliers of stable $C^{*}$-algebras', Trans. Amer. Math. Soc. 299 (1987), 397-411.

[10] N. C. Phillips, Equivariant $K$-theory and freeness of group actions on $C^{*}$-algebras, Lecture Notes in Math. 1274 (Springer, Berlin, 1987).

[11] N. C. Phillips, 'Inverse limits of $C^{*}$-algebras', J. Operator Theory 19 (1988), 159-195.

[12] N. C. Phillips, 'Representable $K$-theory for $\sigma$ - $C^{*}$-algebras', $K$-Theory 3 (1989), $441-$ 478.

[13] N. C. Phillips, 'Classifying algebras for the $K$-theory of $\sigma$ - $C^{*}$-algebras', Canad. $J$. Math. 41 (1989), 1021-1089.

[14] N. C. Phillips, ' $C$ loop algebras and noncommutative Bott periodicity', Trans. Amer. Math. Soc. 325 (1991), 631-659.

[15] N. C. Phillips, ' $K$-theory and noncommutative homotopy theory', in: Operator theory, operator algebras and applications (eds. W. B. Arveson and R. G. Douglas), Proceedings of Symposia in Pure Mathematics 51, part 2 (Amer. Math. Soc., Providence, 1990), pp. 255-265.

[16] N. C. Phillips, ' $K$-theory for Fréchet algebras', International J. Math. 2 (1991), 77-129.

[17] C. Schochet, 'Topological methods for $C^{*}$-algebras III: axiomatic homology', Pacific $J$. Math. 114 (1984), 399-445.

[18] G. Segal, 'The representation ring of a compact Lie group', Publ. Math. Inst. Hautes Études Sci. 34 (1968), 113-128.

[19] J. L. Taylor, 'Banach algebras and topology,' in: Algebras in analysis (Academic Press, London, 1975), pp. 118-186.

[20] J. Weidner, Topological invariants for generalized operator algebras, (Ph.D. Thesis, Heidelberg, 1987).

[21] J. Weidner, ' $K K$-groups for generalized operator algebras I', $K$-Theory 3 (1989), 57-77.

University of Georgia

Athens, GA 30602

USA 